\title{
Pricing of CDO's Based on the Multivariate Wang Transform *
}

\author{
Masaaki Kijima $^{\text {a† }}$, Shin-ichi Motomiya ${ }^{\mathrm{b}}$ and Yoichi Suzuki ${ }^{\mathrm{b}}$ \\ a Tokyo Metropolitan University/Kyoto University \\ b Credit Pricing Corporation, Ltd.
}

(This Version: August 22, 2008)

(First Version: March 15, 2008)

\begin{abstract}
This paper shows that the one-factor Gaussian copula model, the standard market model for valuing CDO's, can be derived from the multivariate Wang transform, which is consistent with Bühlmann's equilibrium pricing model, whence it has a sound economic interpretation. The Gaussian copula model is then extended within the Bühlmann's framework. Unlike the existing models, our model calibrates the parameter associated with risk aversion index of the representative investor, not the correlation parameter. A $t$-copula model is also considered to describe the fat-tail distribution observed in the actual markets.
\end{abstract}

Keywords. One-factor Gaussian copula model, Merton's structural model, Bühlmann's economic premium principle, multivariate Wang transform, Student $t$ distribution.

*A previous version of this paper was presented at the 2nd Risk Management Conference held in Singapore on July 1, 2008. The authors are grateful to participants of the conference for helpful discussions. Numerical assistance by Dr. Yasuhiro Tamba of Credit Pricing Corporation, Ltd. is also acknowledged.

${ }^{\dagger}$ Corresponding author: Graduate School of Social Sciences, Tokyo Metropolitan University, 1-1 Minami-Ohsawa, Hachiohji, Tokyo 192-0397, Japan. TEL:+81-42-677-2330, FAX:+81-42-677-2298, E-mail: kijima@center.tmu.ac.jp 


\section{Introduction}

The one-factor Gaussian copula model, first developed by Li (2000), has become the standard market model for valuing collateralized debt obligations (CDO's) and other basket-type credit derivatives. ${ }^{1}$ The Gaussian copula approach is very convenient to model default time correlation given the marginal default probabilities, and allows the semi-analytical form for the pricing and hedging of such products.

Despite the popularity of the model in practice, however, the copula approach is often criticized due to a couple of reasons. ${ }^{2}$ Among them, it is claimed that the copula approach is difficult to interpret and the dependence structure is exogenously given without a theoretical justification. Also, it is well known that the model cannot explain the market prices of CDO tranches, i.e., it exhibits the so-called correlation smile. ${ }^{3}$ As a result, it cannot price nonstandard credit derivatives such as bespoke CDO's to be consistent with market quotes for tranches of standard CDO's.

The aim of this paper is twofold. First, contrary to the criticism, we show that the one-factor Gaussian copula model is consistent with Bühlmann's equilibrium pricing model (1980), ${ }^{4}$ whence it has a sound economic interpretation. Second, the Gaussian copula model is extended within the Bühlmann's framework to fit market prices of CDO tranches better by taking the well-recognized facts in the credit derivatives market into consideration.

Namely, as in CreditMetrics (1997), we start with Merton's structural model (1974) in which underlying log-firm values follow a one-factor Gaussian copula model under the actual probability measure. ${ }^{5}$ After an appropriate change of measures based on the multivariate

\footnotetext{
${ }^{1}$ See, e.g., Hull and White (2004) and Laurent and Gregory (2005) for details of the one-factor Gaussian copula model.

${ }^{2}$ Given these problems, Mortensen (2006) used a multivariate version of the intensity-based approach to develop a semi-analytical valuation method for CDO's. However, this approach involves many parameters to be estimated and/or calibrated and seems to be computationally difficult and instable to apply for actual markets, compared with the copula approach. See also Duffie and Garleanu (2001) for the intensity-based approach.

${ }^{3}$ If the Gaussian copula model fitted market prices well, the implied compound correlation (base correlation, as well) would be approximately constant across tranches. In order to overcome the deficiency, a number of researchers look for copulas that fit market prices better than the Gaussian copula. See, e.g., Hull and White (2006) and Burtschell, Gregory and Laurent (2007) for such extensions.

${ }^{4}$ Bühlmann's model (1980) has been developed for the pricing and hedging of insurance risk. Insurance market is incomplete, in the sense that risks in the market cannot be replicated by other assets in the market, and so is the CDO market.

${ }^{5}$ CreditMetrics (1997) seems the most popular model to evaluate portfolio credit risk for practitioners. Our model provides a linkage between the credit-risk valuation and the pricing of basket-type credit derivatives.
} 
Wang transform developed by Kijima (2006), we introduce the risk aversion index for each tranche to be calibrated from market quotes for CDO tranches, while keeping the correlation structure as given under the actual probability measure, ${ }^{6}$ since the CDO market is segmented into tranches according to investor's preference against risks. Also, we apply the Student $t$ copula for the risk-adjusted model, because some empirical studies suggest to use $t$ distributions with $\nu=3$ to 7 degrees of freedom for return distributions of financial and insurance products. ${ }^{7}$

This paper is organized as follows. In the next section, we briefly explain the fact that the multivariate Wang transform is consistent with Bühlmann's economic premium principle (1980). It is then shown in Section 3 that the one-factor Gaussian copula model is derived from the multivariate Wang transform, whence it has a sound economic interpretation. Section 4 is devoted to propose an alternative to the standard Gaussian copula model within the Bühlmann's framework. Unlike the existing models, our model calibrates the parameter associated with risk aversion index of the representative investor, not the correlation parameter. Moreover, it is shown that the calibrated parameter always exists and is unique for any market price of CDO's. The proof is given in Appendix A. Section 5 considers a Student $t$ copula to describe the fat-tail distribution observed in the actual markets. Numerical examples show that the $t$-copula model fit market quotes for tranches of standard CDO's better than the existing models in the literature. Section 6 concludes this paper.

Throughout this paper, we shall denote the actual probability measure by $P$ and the risk-neutral probability measure by $Q$. The normal distribution with mean $\mu$ and variance $\sigma^{2}$ is denoted by $N\left(\mu, \sigma^{2}\right)$.

\section{The Multivariate Wang Transform}

In the actuarial literature, there have been developed many probability transforms for pricing financial and insurance risks. Recently, Wang (2000, 2002) proposed a pricing method based on the following transformation from $G(x)$ to $G^{Q}(x)$ :

$$
G^{Q}(x)=\Phi\left[\Phi^{-1}(G(x))+\theta\right]
$$

where $\Phi$ denotes the standard normal cumulative distribution function (CDF for short) and $\theta$ is a constant. The transform is called the Wang transform and the CDF $G^{Q}(x)$ is considered

\footnotetext{
${ }^{6}$ This is consistent with Girsanov's theorem for the Gaussian case in continuous-time that, when changing the actual measure to the risk-neutral measure, the mean is adjusted to represent the risk preference of investors, but the variance-covariance structure is unchanged. See, e.g., Kijima (2002) for details.

${ }^{7}$ See, e.g., Platen and Stahl (2003) and Wang (2004) for such empirical studies.
} 
to be a risk-adjusted CDF under the risk-neutral probability measure $Q$. The mean value evaluated under $G^{Q}(x)$ will define a risk-adjusted "fair value" of risk with CDF $G(x)$ under $P$ at some future time, which can be discounted to time zero using the risk-free interest rate. The parameter $\theta$ is considered to be a risk premium.

The Wang transform not only possesses various desirable properties as a pricing method, but also has a sound economic interpretation. For example, as Wang (2003) observed, the transform (2.1) is consistent with Bühlmann's economic premium principle.

More precisely, Bühlmann (1980) considered risk exchanges among a set of agents. Each agent is characterized by his/her exponential utility function $u_{i}(x)=-\mathrm{e}^{-\lambda_{i} x}, i=1,2, \ldots, n$, and faces a risk of potential loss $X_{i}$. In a pure risk exchange model, Bühlmann (1980) derived the following equilibrium pricing formula for risk $X$ :

$$
\pi(X)=E[\eta X], \quad \eta=\frac{\mathrm{e}^{-\lambda Z}}{E\left[\mathrm{e}^{-\lambda Z}\right]},
$$

for which the expectations exist, where $E$ denotes the expectation operator under $P, Z=$ $\sum_{i=1}^{n} X_{i}$ is the aggregate risk, and $\lambda$ is given by

$$
\lambda^{-1}=\sum_{i=1}^{n} \lambda_{i}^{-1}, \quad \lambda_{i}>0 .
$$

The parameter $\lambda$ is thought of as the risk aversion index of the representative agent in the market.

Unfortunately, however, the actuarial pricing functional is not linear, whence admits an arbitrage opportunity. ${ }^{8}$ In order to develop a linear pricing method while maintaining probability distortions, Kijima (2006) derived a multivariate version of the Wang transform (2.1) from the Bühlmann's equilibrium pricing formula (2.2).

Suppose that the underlying risks are described by an $n$-dimensional random vector, $\left(X_{1}, X_{2}, \ldots, X_{n}\right)$ say. Suppose further that the underlying risks are formulated by a Gaussian copula under $P$. That is, define

$$
Z_{i} \equiv \Phi^{-1}\left[G_{i}\left(X_{i}\right)\right], \quad i=1,2, \ldots, n
$$

where $G_{i}(x)$ is the marginal CDF of $X_{i} \cdot{ }^{9}$ A Gaussian copula assumes that $\left(Z_{1}, Z_{2}, \ldots, Z_{n}\right)$ follows an $n$-variate standard normal distribution with correlation matrix $\boldsymbol{\Sigma}=\left(\rho_{i j}\right)$.

\footnotetext{
${ }^{8}$ See, e.g., Harrison and Kreps (1979) for details. The pricing functional $\pi$ is said to be linear if $\pi(a X+$ $b Y)=a \pi(X)+b \pi(Y)$ for all risks $X, Y$ and constants $a, b$.

${ }^{9}$ Throughout the paper, it is assumed for the sake of simplicity that all the CDF's under consideration are strictly increasing.
} 
Now, suppose that the aggregate risk $Z$ consists of many individual risks $X_{i}$ so that it can be approximated by a normal distribution. Let $Z_{0}=\left(Z-\mu_{Z}\right) / \sigma_{Z}$ be the standardized normal random variable, where $\mu_{Z}=E[Z]$ and $\sigma_{Z}^{2}=V[Z]$ denotes the variance of $Z$ under $P$. Next, suppose that the standard normal variable $Z_{0}$ is related to the Gaussian copula as

$$
Z_{0} \stackrel{\mathrm{d}}{=} \xi+\sum_{i=1}^{n} w_{i} Z_{i}, \quad Z_{i}=\Phi^{-1}\left[G_{i}\left(X_{i}\right)\right]
$$

for some constants $w_{i}$ and a random variable $\xi$, which is independent of $Z_{i}$, where $\stackrel{\mathrm{d}}{=}$ stands for equality in law. Using the assumption (2.3), Kijima (2006) obtained the following multivariate transformation from the Bühlmann's equilibrium pricing formula (2.2):

$$
G^{Q}(\boldsymbol{x})=\Phi_{n: \Sigma}\left(\Phi^{-1}\left[G_{1}\left(x_{1}\right)\right]+\sum_{i=1}^{n} \lambda_{i} \rho_{1 i}, \ldots, \Phi^{-1}\left[G_{n}\left(x_{n}\right)\right]+\sum_{i=1}^{n} \lambda_{i} \rho_{n i}\right),
$$

where $\boldsymbol{x}=\left(x_{1}, \ldots, x_{n}\right)$ and $\Phi_{n: \Sigma}$ denotes the CDF of the $n$-variate standard normal distribution with correlation matrix $\Sigma$. Note that, when $n=1,(2.4)$ coincides with the Wang transform (2.1) since $\rho_{11}=1$.

In particular, when $\left(X_{1}, X_{2}, \ldots, X_{n}\right)$ is normally distributed with correlation matrix $\mathbf{\Sigma}=$ $\left(\rho_{i j}\right)$ under $P$, we have $G_{i}(x)=\Phi\left(\left(x-\mu_{i}\right) / \sigma_{i}\right)$, where $\mu_{i}=E\left[X_{i}\right]$ and $\sigma_{i}^{2}=V\left[X_{i}\right]$. It follows that the multivariate Wang transform (2.4) becomes

$$
G^{Q}(\boldsymbol{x})=\Phi_{n: \Sigma}\left(\frac{x_{1}-\mu_{1}}{\sigma_{1}}+\lambda C_{1}, \ldots, \frac{x_{n}-\mu_{n}}{\sigma_{n}}+\lambda C_{n}\right), \quad C_{i} \equiv \frac{1}{\sigma_{i}} \operatorname{Cov}\left(X_{i}, Z\right),
$$

since

$$
\sum_{j=1}^{n} \lambda_{j} \rho_{j i}=\frac{\lambda}{\sigma_{i}} \operatorname{Cov}\left(X_{i}, Z\right)
$$

Here, $\operatorname{Cov}(X, Z)$ is the covariance between $X$ and $Z, \lambda$ is the risk aversion index, and $Z$ denotes the aggregated market risk. We note that, in the normal case, the transform (2.5) can be derived directly from the Bühlmann's formula (2.2) without the assumption (2.3). ${ }^{10}$

\section{The One-Factor Gaussian Copula Model}

Consider a CDO with an asset pool consisting of $n$ defaultable assets whose default times are denoted by $\tau_{i}, i=1,2, \ldots, n$. Let $N_{i}(t)=1_{\left\{\tau_{i} \leq t\right\}}$ be the default indicator process and $M_{i}$ the loss given default of name $i$. The time- $t$ cumulative loss of the asset pool is then defined by

$$
L(t)=\sum_{i=1}^{n} M_{i} N_{i}(t), \quad 0 \leq t \leq T
$$

\footnotetext{
${ }^{10}$ Kijima (2006) also developed a multivariate version of the Esscher transform and showed that the multivariate Wang transform (2.5) agrees with the Esscher counterpart for the normal case.
} 
where $T$ denotes the maturity of the CDO. Throughout the paper, it is assumed that $M_{i}$ are positive constants.

\subsection{The multivariate Merton model}

In order to model the joint distribution of $\left(\tau_{1}, \tau_{2}, \ldots, \tau_{n}\right)$, we follow the structural model of Merton (1974). That is, consider the firm value $V_{i}$ of name $i$, and assume that default occurs before time $t$, i.e. $\tau_{i} \leq t$, if and only if the firm value $V_{i}$ is less than some default threshold. In other words, denoting $X_{i}=\log V_{i}$, we assume that

$$
\left\{\tau_{i} \leq t\right\}=\left\{X_{i}<x\right\}
$$

for some $x{ }^{11}$ It is easier to model the joint distribution of $\left(X_{1}, X_{2}, \ldots, X_{n}\right)$ rather than that of $\left(\tau_{1}, \tau_{2}, \ldots, \tau_{n}\right)$ directly. This is the basic idea adopted by CreditMetrics (1997) to evaluate the portfolio credit risk.

CreditMetrics (1997) assumes that $\left(X_{1}, X_{2}, \ldots, X_{n}\right)$ follows an $n$-variate standard normal distribution with correlation matrix $\Sigma=\left(\rho_{i j}\right)$. However, it is computationally very time consuming to obtain the distribution for the cumulative loss $L(t)$ given by (3.1) for the general correlation case. Hence, CreditMetrics (1997) employs a Monte Carlo simulation to approximate the distribution for $L(t)$ under the given correlation structure. Note that CreditMetrics (1997) works under the actual measure $P$, not under the risk-neutral probability measure $Q$, for the purpose of credit risk management. ${ }^{12}$

The industry convention to model the joint distribution of $\left(X_{1}, X_{2}, \ldots, X_{n}\right)$ is to employ the one-factor Gaussian model. ${ }^{13}$ More specifically, let $U$ and $U_{i}, i=1,2, \ldots, n$, be independent and suppose that they follow the standard normal distribution $N(0,1)$. Define

$$
X_{i}=\rho_{i} U+\sqrt{1-\rho_{i}^{2}} U_{i}, \quad i=1,2, \ldots, n,
$$

where $-1<\rho_{i}<1$ are constants. It is readily seen that $X_{i}$ also follow $N(0,1)$ with correlation $\rho_{i j}=\rho_{i} \rho_{j}, i \neq j$. In the following, we denote the correlation matrix for this special case by $\boldsymbol{\Sigma}_{\rho}$. Also, from (3.2), we assume that

$$
F_{i}(t)=\Phi(x) \quad \Longleftrightarrow \quad x=\Phi^{-1}\left(F_{i}(t)\right), \quad i=1,2, \ldots, n,
$$

\footnotetext{
${ }^{11}$ Alternatively, as in Black and Cox (1976), the default time is defined as the first hitting time to the threshold. The European counterpart (3.2) will not give identical results to the first hitting model. However, as Baxter (2007) noted, we would be able to calibrate the parameters so that our method matches the barrier-style method closely.

${ }^{12}$ While the actual measure is used for risk management, the risk-neutral measure is needed only for the pricing of financial products. See Kijima and Muromachi (2000) for details.

${ }^{13}$ An extension of the model to the multi-factor case is straightforward.
} 
where $F_{i}(t)$ denotes the marginal CDF of $\tau_{i}$.

Given the common factor $U$ in (3.3), the conditional default probability before time $t$ of name $i$ is obtained as

$$
q_{i}(t \mid U) \equiv P\left\{\tau_{i} \leq t \mid U\right\}=P\left\{X_{i} \leq x \mid U\right\}=\Phi\left(\frac{\Phi^{-1}\left(F_{i}(t)\right)-\rho_{i} U}{\sqrt{1-\rho_{i}^{2}}}\right) .
$$

Since $\tau_{i}$ are conditionally independent, the joint CDF of $\left(\tau_{1}, \tau_{2}, \ldots, \tau_{n}\right)$, denoted by $F(\boldsymbol{t})$, $\boldsymbol{t}=\left(t_{1}, \ldots, t_{n}\right)$, under the actual probability measure $P$ is given by

$$
F(\boldsymbol{t})=\int_{-\infty}^{\infty}\left[\prod_{i=1}^{n} \Phi\left(\frac{\Phi^{-1}\left(F_{i}\left(t_{i}\right)\right)-\rho_{i} u}{\sqrt{1-\rho_{i}^{2}}}\right)\right] \phi(u) \mathrm{d} u
$$

where $\phi(u)$ is the probability density function (PDF for short) of the standard normal distribution $N(0,1)$.

\subsection{Change of measures from $P$ to $Q$}

In this subsection, we apply the change of measure formula (2.5) to obtain the distribution for the cumulative loss $L(t)$ under the risk-neutral probability measure $Q$. Recall that, in Girsanov's theorem for the Gaussian setting in continuous time, while the variance-covariance structure is unchanged, the mean is adjusted to represent the risk preference of investors under the change of measures from $P$ to $Q$.

Consider the multivariate risks $\left(X_{1}, X_{2}, \ldots, X_{n}\right)$, each $X_{i}$ being defined by (3.3) under $P$. We denote the joint CDF of $\left(\tau_{1}, \tau_{2}, \ldots, \tau_{n}\right)$ under $Q$ by $F^{Q}(\boldsymbol{t}), \boldsymbol{t}=\left(t_{1}, t_{2}, \ldots, t_{n}\right)$. Since $X_{i}$ follows $N(0,1)$ under $P$, we have from $(2.5)$ that

$$
G^{Q}(\boldsymbol{x})=\Phi_{n: \Sigma_{\rho}}\left(x_{1}+\lambda C_{1}, \ldots, x_{n}+\lambda C_{n}\right),
$$

where $\boldsymbol{\Sigma}_{\rho}=\left(\rho_{i j}\right)$ with $\rho_{i j}=\rho_{i} \rho_{j}, G^{Q}(\boldsymbol{x})$ is the joint $\operatorname{CDF}$ of $\left(X_{1}, X_{2}, \ldots, X_{n}\right)$ under $Q$, and

$$
C_{i} \equiv \operatorname{Cov}\left(X_{i}, Z\right)=1-\rho_{i}^{2}+\rho_{i} \sum_{j=1}^{n} \rho_{j}
$$

Note that $\left(X_{1}, X_{2}, \ldots, X_{n}\right)$ also follows an $n$-variate normal distribution with means $-\lambda C_{i}$ and the same correlation matrix $\Sigma_{\rho}$ under the risk-neutral probability measure $Q$.

Now, we have from (3.2) and (3.7) that

$$
F_{i}^{Q}(t) \equiv Q\left\{\tau_{i} \leq t\right\}=Q\left\{X_{i} \leq x\right\}=\Phi\left(x+\lambda C_{i}\right), \quad i=1,2, \ldots, n
$$

or, equivalently,

$$
x=\Phi^{-1}\left(F_{i}^{Q}(t)\right)-\lambda C_{i}, \quad i=1,2, \ldots, n .
$$


It follows from (3.7) and (3.9) that the joint $\operatorname{CDF} F^{Q}(\boldsymbol{t})$ of $\left(\tau_{1}, \tau_{2}, \ldots, \tau_{n}\right)$ under the riskneutral measure $Q$ is given by

$$
F^{Q}(\boldsymbol{t})=\Phi_{n: \Sigma_{\rho}}\left(\Phi^{-1}\left(F_{1}^{Q}\left(t_{1}\right)\right), \ldots, \Phi^{-1}\left(F_{n}^{Q}\left(t_{n}\right)\right)\right)
$$

But, given the common factor $U$ in (3.3), the conditional default probability before time $t$ of name $i$ under $Q$ is obtained as

$$
q_{i}^{Q}(t \mid U) \equiv Q\left\{\tau_{i} \leq t \mid U\right\}=Q\left\{X_{i} \leq x \mid U\right\}=\Phi\left(\frac{\Phi^{-1}\left(F_{i}^{Q}(t)\right)-\rho_{i} U}{\sqrt{1-\rho_{i}^{2}}}\right)
$$

where we have used (3.9) in the second equality. The joint $\operatorname{CDF}^{Q}(\boldsymbol{t})$ under $Q$ is then given by

$$
F^{Q}(\boldsymbol{t})=\int_{-\infty}^{\infty}\left[\prod_{i=1}^{n} \Phi\left(\frac{\Phi^{-1}\left(F_{i}^{Q}\left(t_{i}\right)\right)-\rho_{i} u}{\sqrt{1-\rho_{i}^{2}}}\right)\right] \phi(u) \mathrm{d} u .
$$

Hence, assuming $\rho_{i}=\rho$, we recover the one-factor Gaussian copula model, the standard market model for the pricing of CDO's, from Merton's structural model (3.2) and Bühlmann's economic premium principle (2.2).

Note that, in the joint $\operatorname{CDF} F^{Q}(\boldsymbol{t})$, the risk aversion index $\lambda$ is embedded in the marginal default $\operatorname{CDF} F_{i}^{Q}(t)$ given by (3.8), and the joint $\operatorname{CDF} F^{Q}(\boldsymbol{t})$ under $Q$ looks similar to that under $P$; cf. (3.6). The $\mathrm{CDF} F_{i}^{Q}(t)$ can be calibrated from market quotes for, e.g., credit default swaps (CDS's).

The distribution for the cumulative loss $L(t)$ under $Q$ (and also under $P$ ) can be obtained relatively easily. Let $\varphi(s \mid t)$ be the characteristic function of $L(t)$. Since $\tau_{i}$ are conditionally independent given the common factor $U$, we obtain

$$
\varphi(s \mid t) \equiv E^{Q}\left[\mathrm{e}^{i s L(t)}\right]=E^{Q}\left[E^{Q}\left[\mathrm{e}^{i s L(t)} \mid U\right]\right]=E^{Q}\left[\prod_{i=1}^{n} \varphi_{i}(s \mid t, U)\right],
$$

where $E^{Q}$ denotes the expectation operator under $Q$ and the conditional characteristic function $\varphi_{i}(s \mid t, U)$ is given by

$$
\varphi_{i}(s \mid t, U) \equiv E^{Q}\left[\mathrm{e}^{i s M_{i} N_{i}(t)} \mid U\right]=q_{i}^{Q}(t \mid U) \mathrm{e}^{i s M_{i}}+\left(1-q_{i}^{Q}(t \mid U)\right) .
$$

Here, $q_{i}^{Q}(t \mid U)$ is the conditional default probability given by (3.11). The distribution for $L(t)$ can then be numerically inverted back from $\varphi(s \mid t)$ using, e.g., the fast Fourier transform. ${ }^{14}$

\footnotetext{
${ }^{14}$ Alternatively, we can apply the bucketing method developed by Hull and White (2004).
} 


\section{A Risk-Adjusted Gaussian Copula Model}

We have seen that the one-factor Gaussian copula model (3.12) has a sound economic interpretation within the Bühlmann's equilibrium pricing framework. However, it is also very well known that the model cannot explain the market prices of CDO tranches. In this section, we modify the standard model (3.12) by introducing a risk aversion index, called a base lambda, for each tranche of CDO's. The risk aversion indices are calibrated from market quotes for tranches of standard CDO's.

\subsection{Risk adjustment}

It is often said that the CDO market is segmented into tranches according to investor's preference against risks. Hence, it is natural to assume that the risk aversion index differs over tranches. More precisely, for a base tranche with detachment point $D$, which we call tranche $D$ for short, we assume that the risk aversion index is given by $\lambda+\lambda_{D}$. In other words, when evaluating tranche $D$, we assume that $\left(X_{1}, X_{2}, \ldots, X_{n}\right)$ follows an $n$-variate normal distribution with means $-\left(\lambda+\lambda_{D}\right) C_{i}$ and the correlation matrix $\Sigma_{\rho}$ under the riskneutral probability measure $Q$. The parameter $\lambda_{D}$ can be negative, because it represents the risk adjustment for tranche $D$. We call $\lambda_{D}$ the base lambda for tranche $D$.

Recall that the CDF's $F_{i}^{Q}(t)$ are calibrated from market quotes for CDS's. In other words, the default boundary $x$ remains to be the same as in (3.9). It follows from (3.10) that the joint $\operatorname{CDF} F_{D}^{Q}(\boldsymbol{t})$, when evaluating tranche $D$, of default times $\tau_{i}$ under $Q$ is obtained as

$$
F_{D}^{Q}(\boldsymbol{t})=\Phi_{n: \Sigma_{\rho}}\left(\Phi^{-1}\left(F_{1}^{Q}\left(t_{1}\right)\right)+\lambda_{D} C_{1}, \ldots, \Phi^{-1}\left(F_{n}^{Q}\left(t_{n}\right)\right)+\lambda_{D} C_{n}\right)
$$

The associated conditional default probability before time $t$ of name $i$ under $Q$ is given by

$$
q_{i: D}^{Q}(t \mid U) \equiv Q\left\{\tau_{i} \leq t \mid U, D\right\}=\Phi\left(\frac{\Phi^{-1}\left(F_{i}^{Q}(t)\right)+\lambda_{D} C_{i}-\rho_{i} U}{\sqrt{1-\rho_{i}^{2}}}\right)
$$

It follows from (4.1) and (4.2) that

$$
F_{D}^{Q}(\boldsymbol{t})=\int_{-\infty}^{\infty}\left[\prod_{i=1}^{n} \Phi\left(\frac{\Phi^{-1}\left(F_{i}^{Q}\left(t_{i}\right)\right)+\lambda_{D}-\rho_{i} u}{\sqrt{1-\rho_{i}^{2}}}\right)\right] \phi(u) \mathrm{d} u .
$$

Note that, when $\lambda_{D}=0$, the distributions $F_{D}^{Q}(\boldsymbol{t})$ and $q_{i: D}^{Q}(t \mid U)$ are reduced to $F^{Q}(\boldsymbol{t})$ and $q_{i}^{Q}(t \mid U)$, respectively. 


\subsection{Parameter estimation and calibration}

Our model (4.3) involves parameters $\lambda_{D}, \rho_{i}$ and the marginal CDF's $F_{i}^{Q}(t), i=1,2, \ldots, n$ to be estimated or calibrated from market data.

Calibration is necessary for parameters that reflect risk attitude of investors. In our model, this role is taken by the base lambdas $\lambda_{D}$ and the CDF's $F_{i}^{Q}(t)$ of default times $\tau_{i}$ under $Q$. The calibrated lambda is called the implied lambda. Recall that the CDF's $F_{i}^{Q}(t)$ can be calibrated from market quotes for CDS's.

On the other hand, since we start from Merton's structural model (1974) with the assumption that

$$
X_{i}=\log V_{i}=\rho_{i} U+\sqrt{1-\rho_{i}^{2}} U_{i}
$$

it is natural to estimate the correlation parameters $\rho_{i}$ using the conventional regression with the (standardized) stock return $X_{i}$, if name $i$ issues a stock, to the (standardized) return $U$ of the market index.

Given the CDF's $F_{i}^{Q}(t)$ of default times and the correlation parameters $\rho_{i}$, the base lambda $\lambda_{D}$ can be calibrated from market quotes for tranche $D$ of standard CDO's. Nonstandard credit derivatives such as bespoke CDO's are then priced using, e.g., appropriate interpolation of the base lambdas. Appendix A shows that the base lambda $\lambda_{D}$ always exists and is unique for any market price of CDO's. ${ }^{15}$

We note that, for standard copula models such as the one-factor Gaussian copula model, default correlation among the underlying names is the only unobservable element and, as a result, the correlation plays a role of risk aversion of investors. The industry convention for this purpose is to assume that $\rho_{i}=\rho$ for all $i$, and the parameter $\rho$ implied by the market prices, called the implied correlation, is used as in much the same way as implied volatilities for the Black-Scholes model. However, this usage of correlation yields an apparent limitation on the model flexibility, as we shall see below.

\subsection{A numerical example}

In this numerical example, we consider 5 data sets consisting of market quotes for synthetic CDO's and underlying CDS's for the most liquid 5 year maturity. The CDO quotes are available on the five benchmark tranches trading on the Dow Jones iTraxx index, consisting

\footnotetext{
${ }^{15}$ This is one of advantages in our model. In the standard copula approach, the existence of implied correlations is not guaranteed in general. In order to overcome the deficiency, some recent papers such as Baxter (2007) introduce a global catastrophe factor (often called the Armageddon factor) with low intensity. That is, when the Armageddon occurs, all the names included in the asset pool default simultaneously.
} 
of 125 European investment grade companies, with 0-3\%, 3-6\%, 6-9\%, 9-12\% and 12-22\% tranches, and iTraxx Japan index, consisting of 80 Japanese investment grade companies, with the same tranches. All quotes are obtained from Bloomberg.

Figure 1 shows the price dynamics of each tranche of Dow Jones iTraxx index from September 2006 to March 2008. After the collapse of credit derivatives market on July 2007, the CDO prices have risen sharply. Some market quotes for the benchmark tranches are listed in Table 1. Accordingly, implied correlations seem to have become extremely high. ${ }^{16}$ As usual, strong correlation smiles are observed for the data.

On the contrary to this, as Figure 2 reveals, actual correlations between the underlying assets have dropped after the collapse. ${ }^{17}$ Hence, the sharp rise of prices cannot be attributed to the increase of correlations. We should rather think of it as a result of the change in risk attitude of investors. Recall that our model involves risk aversion parameters $\lambda_{D}$ of the representative agent in the market and the correlation structure remains the same after the change of measure.

Now, the base lambdas $\lambda_{D}$ are calibrated from mid-prices by using a simple bisection method (see Proposition A.2). Selected curves of the calibrated base lambdas for Dow Jones iTraxx index are depicted in Figure 3 (see also Table 1). The base lambda curves can take various shapes to represent investor's preference against risks in tranches. In particular, it is interesting to see from Table 1 that, while $\lambda_{D}$ are all positive on July 30, 2007 in iTraxx Japan index, they are all negative on December 14, 2007. This is so, because the CDO spreads were relatively wide compared to the CDS spreads on July 30, 2007, while the CDS spreads became wider compared to the CDO spreads on December 14, 2007. Recall that the parameter $\lambda$ represents the risk aversion index for CDS in our Gaussian copula model, and $\lambda+\lambda_{D}$ does for tranche $D$ of CDO's. The same interpretation for the base lambdas applies for the results in Figure 3.

\section{$5 \quad$ A Risk-Adjusted Model with $t$ Copula}

The risk-adjusted Gaussian copula model (4.3) has an apparent advantage over the existing models, because it can perfectly fit market quotes for all tranches of standard CDO's by

\footnotetext{
${ }^{16}$ The implied compound correlation for the 9-12\% tranche of iTraxx Japan on March 14, 2008 does not exist for the standard one-factor Gaussian copula model.

${ }^{17}$ The curve in Figure 2 shows a simple average of correlation parameters $\rho_{i}$ calculated by the ordinary regression $X_{i}=\rho_{i} U+\sigma_{i} U_{i}$, where $X_{i}$ stands for the stock return of asset $i, U$ the return of market index, and $U_{i}$ an error term.
} 
Figure 1: Price dynamics of tranches of Dow Jones iTraxx index

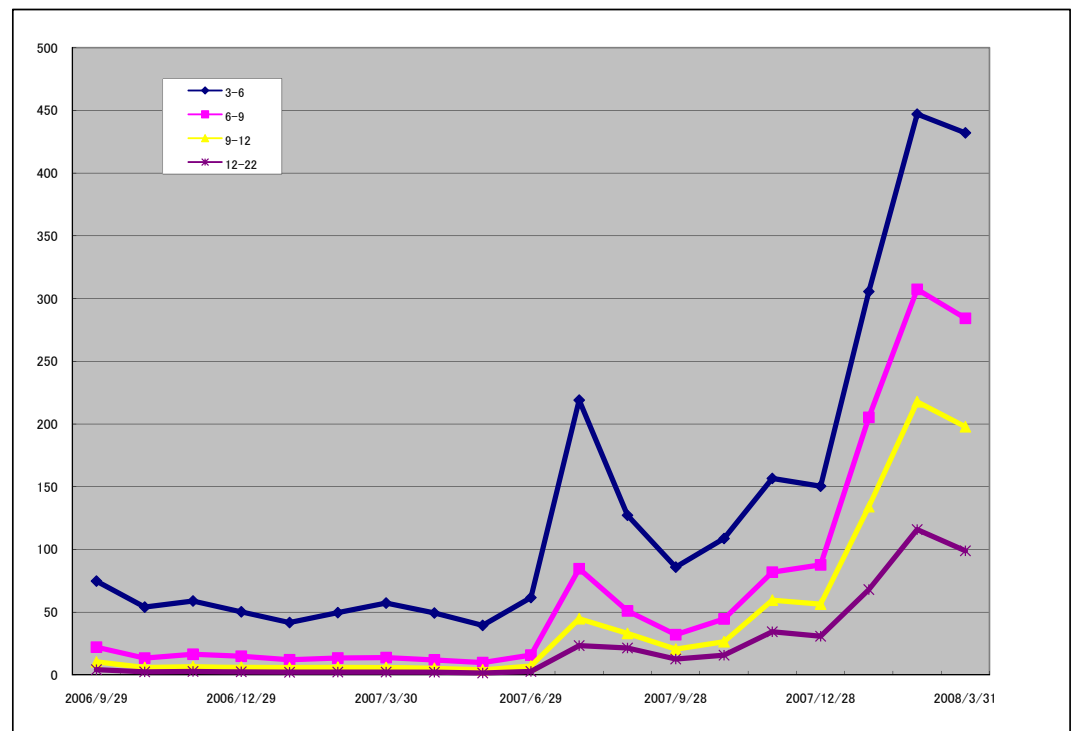

Figure 2: Averaged correlations of underlying assets

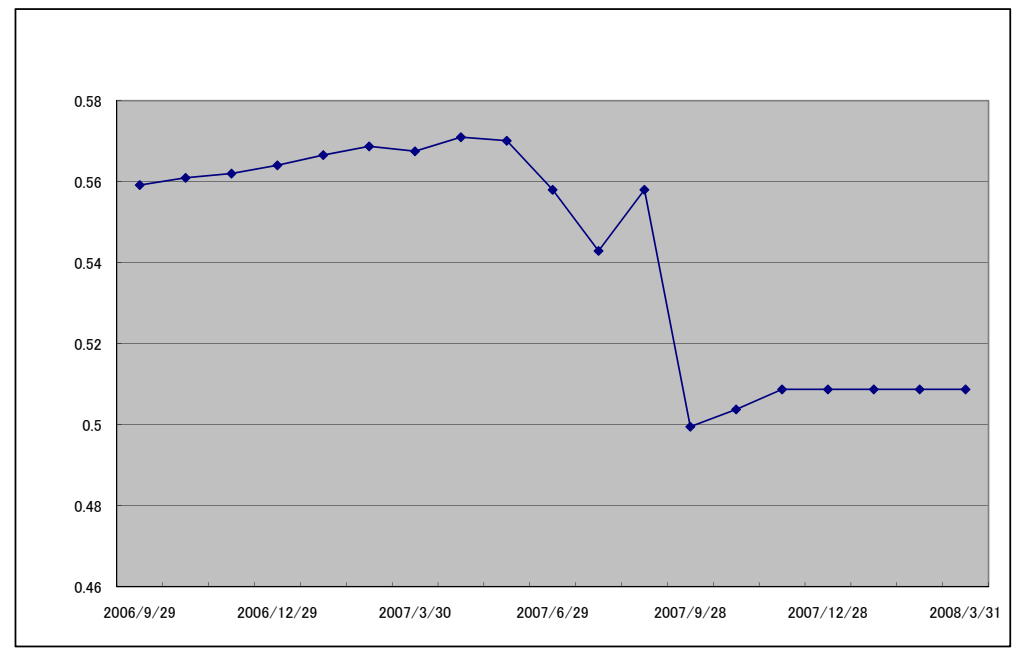

The curve shows an arithmetic average of correlation parameters $\rho_{i}$ calculated by the ordinary regression $X_{i}=\rho_{i} U+\sigma_{i} U_{i}$, where $X_{i}$ stands for the stock return of asset $i, U$ the return of market index, and $U_{i}$ an error term. The regression is performed for monthly data of 5 years. 
Table 1: Market and model prices for CDO tranches

\begin{tabular}{|c||c|c|c|c|c|c|}
\hline Index & Tranches & $0-3 \%$ & $3-6 \%$ & $6-9 \%$ & $9-12 \%$ & $12-22 \%$ \\
\hline DJ iTraxx & Mid Price & $25.5 \%$ & 146.0 & 60.3 & 36.3 & 19.3 \\
$8 / 23 / 2004$ & Bid/Ask Spread & $1.3 \%$ & 10.0 & 5.5 & 5.5 & 3.5 \\
& Calibrated $\lambda_{D}$ & 0.0012 & -0.0009 & -0.0013 & -0.0014 & -0.0016 \\
\hline DJ iTraxx & Mid Price & $26.3 \%$ & 80.6 & 23.1 & 10.3 & 5.8 \\
12/05/2005 & Bid/Ask Spread & $0.6 \%$ & 3.3 & 2.6 & 2.0 & 1.3 \\
& Calibrated $\lambda_{D}$ & 0.0031 & -0.0002 & -0.0012 & -0.0016 & -0.0020 \\
\hline iTraxx Japan & Mid Price & $12.2 \%$ & 40.0 & 11.0 & 6.5 & 3.5 \\
7/05/2007 & Bid/Ask Spread & $1.5 \%$ & 10.0 & 4.0 & 5.0 & 3.0 \\
& Calibrated $\lambda_{D}$ & 0.0023 & -0.0003 & -0.0011 & -0.0014 & -0.0014 \\
\hline iTraxx Japan & Mid Price & $32.0 \%$ & 164.0 & 51.0 & 31.0 & 19.8 \\
7/30/2007 & Bid/Ask Spread & $2.0 \%$ & 20.0 & 10.0 & 10.0 & 2.0 \\
& Calibrated $\lambda_{D}$ & 0.0066 & 0.0032 & 0.0019 & 0.0015 & 0.0016 \\
\hline iTraxx Japan & Mid Price & $24.5 \%$ & 192.5 & 62.5 & 30.5 & 16.5 \\
12/14/2007 & Bid/Ask Spread & $3.5 \%$ & 45.0 & 29.0 & 15.0 & 9.0 \\
& Calibrated $\lambda_{D}$ & -0.0042 & -0.0043 & -0.0046 & -0.0047 & -0.0042 \\
\hline iTraxx Japan & Mid Price & $62.8 \%$ & 1065.0 & 625.0 & 315.0 & 210.0 \\
3/14/2008 & Bid/Ask Spread & $9.0 \%$ & 300.0 & 120.0 & 100.0 & 80.0 \\
& Calibrated $\lambda_{D}$ & -0.0103 & -0.0086 & -0.0077 & -0.0078 & -0.0062 \\
\hline
\end{tabular}

The market prices are obtained from Bloomberg. The base lambdas $\lambda_{D}$ are calibrated from the mid-prices based on the joint CDF given by (4.3). The calibration is performed by a simple bisection method. Interest rates are constant at $3 \%$, and the recovery rate is $40 \%$. 
Figure 3: Selected base lambda curves

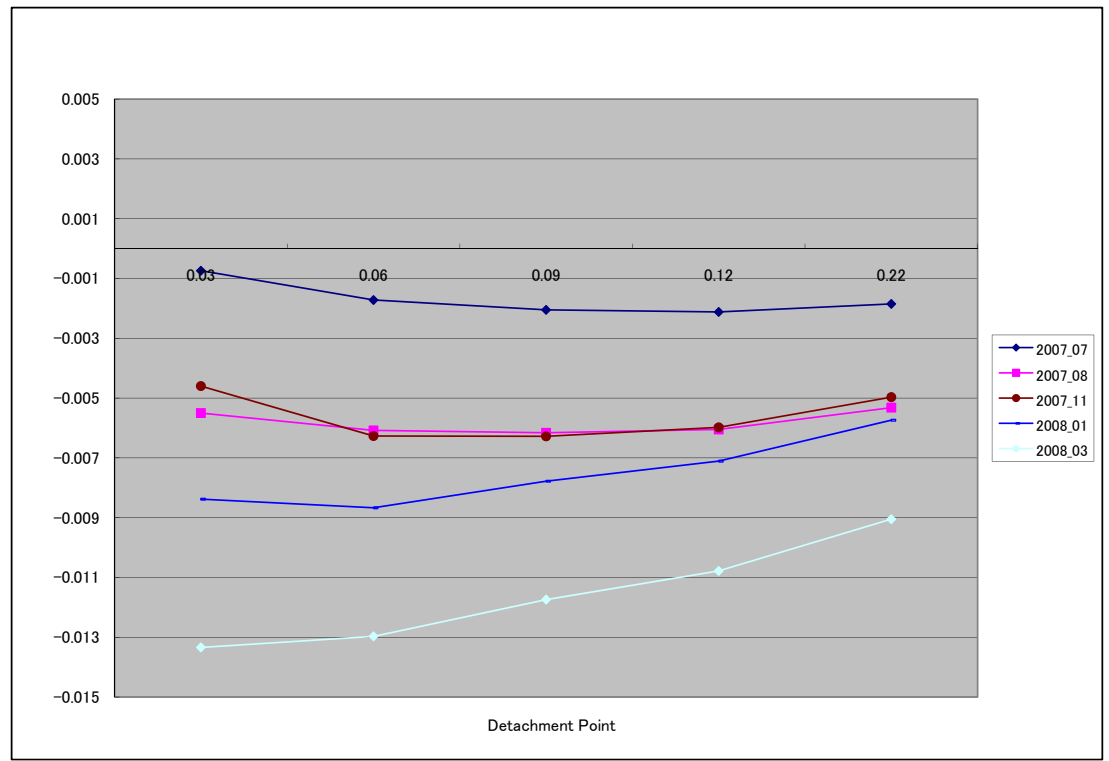

The market prices of benchmark tranches of Dow Jones iTraxx index are obtained from Bloomberg. The base lambdas $\lambda_{D}$ are calibrated from the mid-prices based on the joint CDF given by (4.3). Interest rates are constant at $3 \%$, and the recovery rate is $40 \%$.

calibrating the base lambdas (risk adjustment parameters) $\lambda_{D}$. A non-standard basket credit derivative such as a bespoke CDO can then be priced by using an appropriate base lambda, while keeping the correlation structure as given under $P$. However, the model may be difficult to interpret economically, because we have considered $\lambda+\lambda_{D}$ as the risk aversion index for tranche $D$ of the representative agent in the market. Hence, it makes sense economically to assume that the parameter $\lambda_{D}$ is increasing in $D$, because more risk averse investors will invest higher tranches. For example, a plausible choice for the base lambda $\lambda_{D}$ will be

$$
\lambda_{D}=a+b \log D
$$

and the parameters $a$ and $b$ are to be calibrated. In this section, we consider the Student $t$ copula to the risk-adjusted model (4.1) to overcome this deficiency.

\subsection{Fat-tail distribution}

It is often said that a drawback of the Wang transform (and its extension to the multivariate setting) is the normal CDF $\Phi(x)$ appearing in (2.1) $\left(\Phi_{n: \Sigma}(\boldsymbol{x})\right.$ in (2.4), respectively), that never matches the fat-tailness observed in the actual markets. In fact, some empirical studies suggest to use Student $t$ distributions, whose CDF is denoted by $t_{\nu}(x)$, with $\nu=3$ to 7 degrees 
of freedom for return distributions of financial and insurance assets (see, e.g., Platen and Stahl (2003) and Wang (2004)). In order to overcome this deficiency, Wang (2002) proposed the following two-parameter transformation:

$$
G^{Q}(x)=t_{\nu}\left[\Phi^{-1}(G(x))+\theta\right], \quad \theta>0,
$$

and reported that (5.2) is much better to fit, although the two-parameter transform is not consistent with the economic premium principle (2.2) (see Kijima and Muromachi (2008)).

In our multivariate setting, we also adopt this idea to the joint CDF (4.1) and propose the following multivariate extension of the two-parameter Wang transform: ${ }^{18}$

$$
F_{D}^{Q}(\boldsymbol{t})=t_{n: \nu, \Sigma_{\rho}}\left(\Phi^{-1}\left(F_{1}^{Q}\left(t_{1}\right)\right)+\lambda_{D} C_{1}, \ldots, \Phi^{-1}\left(F_{n}^{Q}\left(t_{n}\right)\right)+\lambda_{D} C_{n}\right)
$$

where $t_{n: \nu, \Sigma_{\rho}}(\boldsymbol{x})$ denotes the CDF of the $n$-variate standard $t$ distribution with $\nu$ degrees of freedom and correlation matrix $\Sigma_{\rho}$ for the underlying standard normal random variables.

Remark 5.1 Hull and White (2004) suggested to use the $t$ distribution for $U$ and $U_{i}$ in (3.3) under the risk-neutral measure $Q$, and reported that the model fits to the market data very well. The good performance of the model is reported in other papers as well. See, e.g., Burtschell, Gregory and Laurent (2005) for details. In this model, however, explicit form for the CDF of $X_{i}$ is not known.

The joint CDF (5.3) can be expressed as follows. Let the default boundary $x$ be given by (3.9), and define the transformed risks $X_{i}^{*}$ as

$$
X_{i}^{*}=\frac{X_{i}}{Y(\nu)}-\left(\lambda+\lambda_{D}\right) C_{i}, \quad Y(\nu)=\sqrt{\chi_{\nu}^{2} / \nu}
$$

where $X_{i}$ are given by (3.3) and $\chi_{\nu}^{2}$ denotes a random variable that follows the chi-square distribution with $\nu$ degrees of freedom, independent of other random variables. It is readily checked that the joint $\mathrm{CDF}$ (5.3) of default times $\tau_{i}$ is given by

$$
F_{D}^{Q}(\boldsymbol{t})=Q\left\{X_{1}^{*} \leq x_{1}, X_{2}^{*} \leq x_{2}, \ldots, X_{n}^{*} \leq x_{n}\right\}
$$

\footnotetext{
${ }^{18}$ Alternatively, we can apply this idea to the joint CDF (3.7). However, the resulting joint CDF of default times $\tau_{i}$ is

$$
F_{D}^{Q}(\boldsymbol{t})=t_{n: \nu, \Sigma_{\rho}}\left(t_{\nu}^{-1}\left(F_{1}^{Q}\left(t_{1}\right)\right)+\lambda_{D} C_{1}, \ldots, t_{\nu}^{-1}\left(F_{n}^{Q}\left(t_{n}\right)\right)+\lambda_{D} C_{n}\right)
$$

which does not have a fatter tail than the Gaussian counterpart. In fact, Kijima and Muromachi (2008) found from numerical experiments that the risk-adjusted distribution has a fatter tail when the inside distribution is less fat-tailed. That is, the two-parameter transformation (5.3) is justified for practical use to represent a fat-tail risk-adjusted distribution.
} 
Now, given the common factor $U$ in (3.3) and $Y(\nu)$ in (5.4), the conditional default probability before time $t$ of name $i$ under $Q$ is obtained as

$$
\begin{aligned}
q_{i: D}^{Q}(t \mid U, Y(\nu)) & \equiv Q\left\{\tau_{i} \leq t \mid U, Y(\nu), D\right\}=Q\left\{X_{i}^{*} \leq x \mid U, Y(\nu)\right\} \\
& =Q\left\{\rho_{i} U+\sqrt{1-\rho_{i}^{2}} U_{i} \leq\left(\Phi^{-1}\left(F_{i}^{Q}(t)\right)+\lambda_{D} C_{i}\right) Y(\nu) \mid U, Y(\nu)\right\} \\
& =\Phi\left(\frac{\left(\Phi^{-1}\left(F_{i}^{Q}(t)\right)+\lambda_{D} C_{i}\right) Y(\nu)-\rho_{i} U}{\sqrt{1-\rho_{i}^{2}}}\right) .
\end{aligned}
$$

Accordingly, the joint $\mathrm{CDF}_{D}^{Q}(\boldsymbol{t})$ under the risk-neutral measure $Q$ is given by

$$
F_{D}^{Q}(\boldsymbol{t})=\int_{-\infty}^{\infty} \int_{0}^{\infty}\left[\prod_{i=1}^{n} \Phi\left(\frac{\left(\Phi^{-1}\left(F_{i}^{Q}\left(t_{i}\right)\right)+\lambda_{D} C_{i}\right) y-\rho_{i} u}{\sqrt{1-\rho_{i}^{2}}}\right)\right] \chi_{\nu}(y) \mathrm{d} y \phi(u) \mathrm{d} u
$$

where $\chi_{\nu}(y)$ is the PDF of $Y(\nu)$.

\subsection{Single integral formula}

In order to avoid the double integral in $(5.6),{ }^{19}$ we employ the following approximation instead of (5.4):

$$
X_{i}^{*}=\frac{X_{i}}{Y_{i}(\nu)}-\left(\lambda+\lambda_{D}\right) C_{i}, \quad Y_{i}(\nu)=\sqrt{\chi_{i: \nu}^{2} / \nu}
$$

where $\chi_{i: \nu}^{2}$ are independent, identically distributed chi-square random variables with $\nu$ degrees of freedom. Then, we can consider the conditional default probability (5.5) to be given by the common factor $U$ only.

More specifically, we define random variables

$$
\xi_{i}(u)=\frac{U_{i}+\delta_{i}(u)}{Y_{i}(\nu)}, \quad \delta_{i}(u)=\frac{\rho_{i} u}{\sqrt{1-\rho_{i}^{2}}}, \quad i=1,2, \ldots, n .
$$

It is well known that $\xi_{i}(u)$ follows a non-central $t$ distribution with $\nu$ degrees of freedom and non-centrality parameter $\delta_{i}(u)$. In the following, we denote the CDF of $\xi_{i}(u)$ by $P_{\nu: \delta(u)}(x)$.

Now, given the common factor $U$ in (3.3), we have from (5.7) and (5.8) that

$$
\left\{X_{i}^{*}<x\right\}=\left\{\xi_{i}(U)<\frac{x+\left(\lambda+\lambda_{D}\right) C_{i}}{\sqrt{1-\rho_{i}^{2}}}\right\},
$$

where $x$ is defined by (3.9). It follows that, given the common factor $U$, the conditional default probability before time $t$ of name $i$ under $Q$ is obtained as

$$
q_{i: D}^{Q}(t \mid U) \equiv Q\left\{\tau_{i} \leq t \mid U, D\right\}=Q\left\{X_{i}^{*}<x \mid U\right\}
$$

\footnotetext{
${ }^{19}$ The double integral is computationally time consuming when the parameters are calibrated to the market data. We aim to derive a simple model for practical use.
} 


$$
\begin{aligned}
& =Q\left\{\xi_{i}(U)<\frac{\Phi^{-1}\left(F_{i}^{Q}(t)\right)+\lambda_{D} C_{i}}{\sqrt{1-\rho_{i}^{2}}} \mid U\right\} \\
& =P_{\nu: \delta_{i}(U)}\left(\frac{\Phi^{-1}\left(F_{i}^{Q}(t)\right)+\lambda_{D} C_{i}}{\sqrt{1-\rho_{i}^{2}}}\right) .
\end{aligned}
$$

The joint $\operatorname{CDF} F_{D}^{Q}(\boldsymbol{t})$ of default times $\tau_{i}$, when evaluating tranche $D$, under the risk-neutral measure $Q$ is then given by

$$
\begin{aligned}
F_{D}^{Q}(\boldsymbol{t}) & =\int_{-\infty}^{\infty}\left[\prod_{i=1}^{n} P_{\nu: \delta_{i}(u)}\left(\frac{\Phi^{-1}\left(F_{i}^{Q}\left(t_{i}\right)\right)+\lambda_{D} C_{i}}{\sqrt{1-\rho_{i}^{2}}}\right)\right] \phi(u) \mathrm{d} u, \\
\delta_{i}(u) & =\frac{\rho_{i} u}{\sqrt{1-\rho_{i}^{2}}} .
\end{aligned}
$$

The distribution for the cumulative loss $L(t)$ under $Q$ can be obtained by using, e.g., the bucketing algorithm of Hull and White $(2004) .{ }^{20}$

\subsection{Empirical application}

The model (5.10) involves new parameters $a$ and $b$ in (5.1) and $\nu$, the degree of freedom in the Student $t$ distribution. Given the marginal CDF's $F_{i}^{Q}(t)$ and the correlation parameters $\rho_{i}$, these new parameters are calibrated from market quotes for CDO tranches by minimizing the following root mean square price errors (RMSE) relative to bid/ask spreads:

$$
\mathrm{RMSE}=\sqrt{\frac{1}{5} \sum_{j=1}^{5}\left(\frac{S_{j: \mathrm{mid}}-S_{j}}{S_{j: \mathrm{ask}}-S_{j: \mathrm{bid}}}\right)^{2}},
$$

where $S_{j}$ is the price of tranche $j$ calculated by the model, $S_{j \text { :mid }}$ the market mid-price, $S_{j \text { :ask }}$ the market ask price, and $S_{j: \text { bid }}$ the market bid price; see Mortensen (2006) for details.

The calibrated results are shown in Tables 2 and 3, where the calibration results for other models are taken from Mortensen (2006). Our risk-adjusted $t$-copula model fits the market prices very well, or at least comparable with the existing models. It is interesting to note that the mid price in equity tranche on December 5, 2005 is higher than that on August 23, 2004, while the mid prices in higher tranches on December 5, 2005 are smaller than those on August 23, 2004. In our model, this tranche structure of mid prices is captured by the degree of freedom $\nu$ of $t$ distribution as well as the slope $b$ of the base lambda $\lambda_{D}$ defined in (5.1). More specifically, the slope $b$ on December 5, 2005 is steeper than that on August 23, 2004, whereas the degree of freedom $\nu$ on December 5, 2005 is bigger than that on August $23,2004$.

\footnotetext{
${ }^{20}$ The CDF $P_{\nu: \delta_{i}}(x)$ can be evaluated easily with enough accuracy by using the algorithm developed in Lenth (1989). See Kijima and Muromachi (2008) for details.
} 
Table 2: Comparison of calibration results for DJ iTraxx index on August 23, 2004

\begin{tabular}{|c||c|c|c|c|c||c|}
\hline Tranches & $0-3 \%$ & $3-6 \%$ & $6-9 \%$ & $9-12 \%$ & $12-22 \%$ & RMSE \\
\hline Mid Price & $25.5 \%$ & 146.0 & 60.3 & 36.3 & 19.3 & \\
Bid/Ask Spread & $1.3 \%$ & 10.0 & 5.5 & 5.5 & 3.5 & \\
\hline Jump-diffusion intensities & $25.0 \%$ & 145.1 & 58.6 & 38.1 & 17.7 & 0.34 \\
Pure diffusion intensities & $30.0 \%$ & 187.1 & 27.4 & 3.5 & 0.1 & 5.11 \\
Gaussian copula & $27.4 \%$ & 222.3 & 52.5 & 13.8 & 1.6 & 4.58 \\
EFL Gaussian copula & $25.3 \%$ & 148.9 & 52.4 & 43.4 & 17.9 & 0.90 \\
Double $t$ copula & $24.0 \%$ & 153.4 & 56.5 & 32.4 & 16.4 & 0.84 \\
\hline Risk adjusted $t$ copula & $25.8 \%$ & 145.2 & 49.1 & 28.4 & 17.1 & 1.15 \\
\hline
\end{tabular}

Calibrated parameters: $\quad \nu=3 \quad \lambda_{D}=0.0045 \times \log D-0.0372$

Table 3: Comparison of calibration results for DJ iTraxx index on December 5, 2005

\begin{tabular}{|c||c|c|c|c|c||c|}
\hline Tranches & $0-3 \%$ & $3-6 \%$ & $6-9 \%$ & $9-12 \%$ & $12-22 \%$ & RMSE \\
\hline Mid Price & $26.3 \%$ & 80.6 & 23.1 & 10.3 & 5.8 & \\
Bid/Ask Spread & $0.6 \%$ & 3.3 & 2.6 & 2.0 & 1.3 & \\
\hline Jump-diffusion intensities & $28.7 \%$ & 86.3 & 18.7 & 14.4 & 10.4 & 2.88 \\
Pure diffusion intensities & $32.5 \%$ & 104.3 & 8.9 & 0.8 & 0.0 & 6.99 \\
Gaussian copula & $34.6 \%$ & 99.9 & 2.9 & 0.1 & 0.0 & 8.44 \\
EFL Gaussian copula & $27.0 \%$ & 83.2 & 9.4 & 7.4 & 7.3 & 2.54 \\
Double $t$ copula & $29.8 \%$ & 101.1 & 24.4 & 13.2 & 6.6 & 3.99 \\
\hline Risk adjusted $t$ copula & $26.5 \%$ & 77.2 & 18.5 & 12.6 & 8.3 & 1.37 \\
\hline
\end{tabular}

Calibrated parameters: $\quad \nu=1 \quad \lambda_{D}=0.024 \times \log D-0.3675$

The market prices were obtained from Bloomberg. The results in the row 'Risk adjusted $t$ copula' are calibrated from the market quotes based on the joint CDF given by (5.10). The results in the other rows are taken from Mortensen (2006). Interest rates are constant at 3\%, and the recovery rate is $40 \%$. 


\section{Conclusion}

This paper showed that, contrary to the criticism, the one-factor Gaussian copula model has a sound economic interpretation, because it is consistent with Bühlmann's equilibrium pricing model. Based on this finding, we then develop an alternative within the Bühlmann's framework to evaluate CDO's and other basket-type credit derivatives. Unlike the existing models, our model calibrates the parameter associated with risk aversion index of the representative investor, not the correlation parameter. More precisely, we introduce the risk aversion index, called the base lambda, for each tranche to be calibrated from market quotes for CDO tranches, while keeping the correlation structure as given under the actual probability measure, since the CDO market is segmented into tranches according to investor's preference against risks.

We also consider a Student $t$ copula within the Bühlmann's framework, because some empirical studies suggest to use $t$ distributions with $\nu=3$ to 7 degrees of freedom for return distributions of financial and insurance assets. Numerical experiments reveal that our model provide a better fit than the existing models in the literature.

\section{A Existence and Uniqueness of the Base Lambda}

For random variables $X$ and $Y, X$ is said to be greater than $Y$ in the sense of first-order stochastic dominance (FSD for short), written by $X \geq_{\mathrm{FSD}} Y$, if $F_{X}(x) \leq F_{Y}(x)$ for all $x \in R$, where $F_{X}(x)$ and $F_{Y}(x)$ denote the CDF's of $X$ and $Y$, respectively. It is well known that $X \geq_{\text {FSD }} Y$ if and only if $E[f(X)] \geq E[f(Y)]$ for all increasing functions $f$ for which expectations exist. See, e.g., Kijima and Ohnishi (1996) for details of stochastic dominance relations.

Proposition A.1 Suppose that the joint CDF of default times $\tau_{i}$ under $Q$ is given by (4.1). If all $C_{i}=\operatorname{Cov}\left(X_{i}, Z\right)$ are positive, then the cumulative loss $L(t)$ given by (3.1) is increasing with respect to $\lambda_{D}$ in the sense of FSD for each $t$.

Proof. First, suppose that $\tau^{a} \geq_{\mathrm{FSD}} \tau^{b}$. Then, since $N^{a}(t)=1_{\left\{\tau^{a} \leq t\right\}}$, we have

$$
Q\left\{N^{a}(t)=1\right\}=Q\left\{\tau^{a} \leq t\right\} \leq Q\left\{\tau^{b} \leq t\right\}=Q\left\{N^{b}(t)=1\right\}
$$

whence $N^{b}(t) \geq_{\text {FSD }} N^{a}(t)$ for all $t>0$. Second, from (4.2), it is readily seen that, given the common factor $U$, each default time $\tau_{i}$ is decreasing with respect to $\lambda_{D}$ in the sense of FSD. It follows that, given $U$, each default indicator $N_{i}(t)$ is increasing with respect to $\lambda_{D}$ in the 
sense of FSD. But, since FSD satisfies both the convolution and multiplication properties, ${ }^{21}$ it is easy to see that, given the common factor $U$, the cumulative loss $L(t)=\sum_{i=1}^{n} M_{i} N_{i}(t)$ is increasing with respect to $\lambda_{D}$ in the sense of FSD for each $t$. The result follows at once by unconditioning on $U$.

For tranche $[A, D]$, define

$$
h(L)=(L-A)_{+}-(L-D)_{+}, \quad(x)_{+}=\max \{x, 0\} .
$$

The market convention to calculate the price $S$ of the tranche is the following. Let $d(t)$ be the discount function, and let $t_{j}=j / f, j=1,2, \ldots, T f$, where $T$ is the maturity of the CDO and $f$ is the frequency of coupon payments. Further, define

$$
V \equiv \frac{S}{f} \sum_{j=1}^{T f} d\left(t_{j}-\hat{f}\right)\left\{D-A-\frac{1}{2} E^{Q}\left[h\left(L\left(t_{j}\right)\right)+h\left(L\left(t_{j-1}\right)\right)\right]\right\}
$$

and

$$
P \equiv \sum_{j=1}^{T f} d\left(t_{j}-\hat{f}\right) E^{Q}\left[h\left(L\left(t_{j}\right)\right)-h\left(L\left(t_{j-1}\right)\right)\right]
$$

with $L(0)=0$, where $\hat{f}=(2 f)^{-1}$. The price $S$ of the tranche is obtained by solving $V=P$. See, e.g., Mortensen (2006) for details.

Now, the function $h(L)$ in (A.1) is increasing in $L$. It follows from Proposition A.1 that the expectation $E^{Q}\left[h\left(L\left(t_{j}\right)\right)+h\left(L\left(t_{j-1}\right)\right)\right]$ is increasing in $\lambda_{D}$ under the condition of the proposition. Also, (A.3) can be rewritten as

$$
P=\sum_{j=1}^{T f}\left\{d\left(t_{j}-\hat{f}\right)-d\left(t_{j+1}-\hat{f}\right)\right\} E^{Q}\left[h\left(L\left(t_{j}\right)\right)\right],
$$

where we set $d\left(t_{T f+1}-\hat{f}\right)=0$. Hence, while $V$ in (A.2) is decreasing in $\lambda_{D}, P$ in (A.3) is increasing in $\lambda_{D}$ under the condition of Proposition A.1.

Note that, from (4.2), when $\lambda_{D} \rightarrow-\infty$, we have $Q\left\{\tau_{i} \leq t \mid U\right\} \rightarrow 0$ for all $t>0$ so that $\tau_{i} \rightarrow \infty$ and $L(t) \rightarrow 0$. On the other hand, when $\lambda_{D} \rightarrow \infty$, we have $Q\left\{\tau_{i} \leq t \mid U\right\} \rightarrow 1$ for all $t>0$ so that $\tau_{i} \rightarrow 0$ and $L(t) \rightarrow L \equiv \sum_{i=1}^{n} M_{i}$. It follows that, as $\lambda_{D} \rightarrow-\infty$, we have $V \rightarrow \frac{(D-A) S}{f} \sum_{j=1}^{T f} d\left(t_{j}-\hat{f}\right)$ and $P \rightarrow 0$, while $V \rightarrow 0$ and $P \rightarrow d\left(t_{1}-\hat{f}\right)(D-A)$ as $\lambda_{D} \rightarrow \infty$.

In particular, for the base tranche $D$ with $A=0$, we have from (A.1) that

$$
h(L)=\min \{D, L\}, \quad D-h(L)=(D-L)_{+}
$$

\footnotetext{
${ }^{21}$ Suppose $X_{1} \geq$ FSD $Y_{1}, X_{2} \geq$ FSD $Y_{2}$ and the random variables are independent of each other. The convolution property implies that $X_{1}+X_{2} \geq_{\mathrm{FSD}} Y_{1}+Y_{2}$, whereas the multiplication property means $w X_{1} \geq_{\mathrm{FSD}} w Y_{1}$ for $w>0$. Hence, these properties ensure that $w_{1} X_{1}+w_{2} X_{2} \geq_{\text {FSD }} w_{1} Y_{1}+w_{2} Y_{2}$ for $w_{1}, w_{2}>0$.
} 
It follows from (A.2) and (A.4) that

$$
V_{D} \equiv \frac{S}{2 f} \sum_{j=1}^{T f} d\left(t_{j}-\hat{f}\right) E^{Q}\left[\left(D-L\left(t_{j}\right)\right)_{+}+\left(D-L\left(t_{j-1}\right)\right)_{+}\right]
$$

and

$$
P_{D} \equiv \sum_{j=1}^{T f}\left\{d\left(t_{j}-\hat{f}\right)-d\left(t_{j+1}-\hat{f}\right)\right\} E^{Q}\left[\min \left\{D, L\left(t_{j}\right)\right\}\right],
$$

respectively. In this case, as above, $V_{D}$ is decreasing in $\lambda_{D}$ and $P_{D}$ is increasing in $\lambda_{D}$. Also, as $\lambda_{D} \rightarrow-\infty$, we have $V_{D} \rightarrow \frac{D S}{f} \sum_{j=1}^{T f} d\left(t_{j}-\hat{f}\right)$ and $P_{D} \rightarrow 0$, while $V_{D} \rightarrow 0$ and $P_{D} \rightarrow d\left(t_{1}-\hat{f}\right) D$ as $\lambda_{D} \rightarrow \infty$. Therefore, we have proved the following.

Proposition A.2 Suppose that $D<\sum_{i=1}^{n} M_{i}$. Then, the base lambda $\lambda_{D}$ always exists and is unique under the condition of Proposition A.1.

\section{References}

[1] Baxter, M. (2007), "Lévy simple structural model," International Journal of Theoretical and Applied Finance, 10, 593-606.

[2] Black, F. and J. Cox (1976), "Valuing corporate securities: Some effects on bond indenture provisions," Journal of Finance, 31, 351-367.

[3] Burtschell, X., J. Gregory and J.P. Laurent (2005), "A comparative analysis of CDO pricing models," Working Paper, BNP Pariba.

[4] Burtschell, X., J. Gregory and J.P. Laurent (2007), "Beyond the Gaussian copula: Stochastic and local correlation," Journal of Credit Risk, 3, 31-62.

[5] Bühlmann, H. (1980), “An economic premium principle," ASTIN Bulletin, 11, 52-60.

[6] CreditMetrics (1997), JP Morgan.

[7] Duffie, D. and N. Garleanu (2001), "Risk and valuation of collateralized debt obligations," Financial Analyst Journal, Jan/Feb, 41-59.

[8] Harrison, M.J. and D. Kreps (1979), "Martingales and arbitrage in multiperiod securities market," Journal of Economic Theory, 20, 381-408.

[9] Hull, J. and A. White (2004), "Valuation of a CDO and an $n$th to default CDS without Monte Carlo simulation," Journal of Derivatives, 12, 8-23.

[10] Hull, J. and A. White (2006), "Valuing credit derivatives using an implied copula approach," Journal of Derivatives, 14, 8-28.

[11] Kijima, M. (2002), Stochastic Processes with Applications to Finance, Chapman \& Hall, London.

[12] Kijima, M. (2006), "A multivariate extension of equilibrium pricing transforms: The multivariate Esscher and Wang transforms for pricing financial and insurance risks," ASTIN Bulletin, 36, 269-283. 
[13] Kijima, M. and Y. Muromachi (2000), "Evaluation of credit risk of a portfolio with stochastic interest rate and default processes," Journal of Risk, 3, 5-36.

[14] Kijima, M. and Y. Muromachi (2008), "An extension of the Wang transform derived from Bühlmann's economic premium principle for insurance risk," Insurance: Mathematics and Economics, 42, 887-896.

[15] Kijima, M. and M. Ohnishi (1996), "Portfolio selection problems via the bivariate characterization of stochastic dominance relations," Mathematical Finance, 6, 237-277.

[16] Lenth, R.V. (1989), "Cumulative distribution function of the non-central $t$ distribution: Algorithm AS 243," Applied Statistics, 38, 185-189.

[17] Laurent, J.P. and J. Gregory (2005), "Basket default swaps, CDO's and factor copulas," Journal of Risk, 7, 103-122.

[18] Li, D.X. (2000), "On default correlation: A copula function approach," Journal of Fixed Income, 9, 43-54.

[19] Merton, R.C. (1974), "On the pricing of corporate debt: The risk structure of interest rates," Journal of Finance, 29, 449-470.

[20] Mortensen, A. (2006), "Semi-analytical valuation of basket credit derivatives in intensity-based models," Journal of Derivatives, 14, 8-26.

[21] Platen, E. and G. Stahl (2003), "A structure of general and specific market risk," Working paper, University of Technology, Sydney.

[22] Wang, S.S. (2000), "A class of distortion operators for pricing financial and insurance risks," Journal of Risk and Insurance, 67, 15-36.

[23] Wang, S.S. (2002), "A universal framework for pricing financial and insurance risks," ASTIN Bulletin, 32, 213-234.

[24] Wang, S.S. (2003), "Equilibrium pricing transforms: New results using Bühlmann's 1980 economic model," ASTIN Bulletin, 33, 57-73.

[25] Wang, S.S. (2004), "Cat bond pricing using probability transforms," Special Issues on Insurance and the State of the Art in Cat Bond Pricing, 19-29. 